\title{
Stochastic behavior of a cold standby system with maximum repair time
}

\author{
Ashish Kumar*, Sonali Baweja and Monika S. Barak
}

Department of Mathematics, Manipal University Jaipur, Jaipur-303007, Raj, India

\begin{tabular}{l}
\hline C H R O N I C L E \\
\hline Article history: \\
Received January 29, 2015 \\
Received in revised format: \\
April 28, 2015 \\
Accepted May 7, 2015 \\
Available online \\
May 82015 \\
\hline Keywords: \\
Cold standby system \\
Preventive maintenance \\
Priority \\
Maximum operation and repair \\
times
\end{tabular}

\section{Introduction}

Economic growth of a country totally depends on its industrial and mechanical development and this growth gives emergence to new technologies. These advance technologies developed many complex industrial systems with the composition of many simultaneous components. Complexity of the system also affects its performance, cost and reliability. But, consumers expect highly reliable systems at minimum cost. Now, system designers and researchers face a great challenge to develop such systems which operate continually and increase their performance, reliability and safety. Many researchers such as Moghaddass et al. (2011), Zhang and Wang (2009) and Wu and Wu (2011) analysed reliability of repairable systems under different assumptions. Goel and Sharma (1989), Cao and Wu (1989), Chandrasekhar et al. (2004), Gopalan and Nagarwall (1985), Gopalan and Bhanu (1995), Chander (2005), Mahmoud and Moshref (2010), Mokaddis et al. (2008) and Kumar and Malik (2012) discussed several stochastic model under common assumptions that after a maximum operation time unit

\footnotetext{
* Corresponding author.

E-mail address: ashish.kumar@jaipur.manipal.edu (A. Kumar) 
undergoes for preventive maintenance either standby unit available or not. Mokaddis et al. (2008) studied the effect of preventive maintenance on the systems of non-identical units. Jin et al. (2009) discussed option model for joint production and preventive maintenance. But, keeping in mind the economic aspects of the system sometimes this assumption is not practically feasible. It is also proved that preventive maintenance can slow the deterioration process of a repairable system and restore the system in a younger age or state. Malik and Nandal (2010) carried out the cost-benefit analysis of a stochastic model using the concept of preventive maintenance. Osaki and Asakura (1970) and Kapur and Kapoor (1974) developed reliability model for two-unit standby redundant system with repair and preventive maintenance. Thus, the method of preventive maintenance can be used to improve the reliability and profit of system. Nakagawa and Osaki (1975) analysed stochastic behaviour of a twounit parallel redundant system with preventive maintenance.

Further, it is a known fact that frequency of failure of standby systems can be reduced up to a desired extent by the method of redundancy. Therefore, keeping a system in cold standby has attracted the attention of many researchers. Barak and Malik (2014), Kumar and Malik (2015), Kumar et al. (2012) and Barak and Barak (2013) suggested some reliability models using the concept of redundancy. Recently, Malik and Munday (2014) developed stochastic model for a computer system by using the concept of hardware redundancy.

Further, the reliability of a system can be increased by making replacement of the components by new one in case repair time is too long i.e., if it extends to a pre-specific time. Singh and Agrafiotis (1995), Malik and Gitanjali (2012) Malik and Kumar (2011) and Kumar and Malik (2014) analyzed stochastically two-unit cold standby systems subject to maximum operation and repair time.

In view of the above importance and practical application of cold standby systems, a stochastic model is developed here by using the concept of preventive maintenance, priority and maximum repair time. For this purpose, a stochastic model is developed in which initially one unit is operative and other is kept as cold standby. There is a single server who visits the system immediately as and when required. The server takes the unit under preventive maintenance after a maximum operation time at normal mode if one standby unit is available for operation. If the repair of the failed unit is not possible up to a maximum repair time failed unit is replaced by new one. The failure time, maximum operation time and maximum repair time distributions of the unit are considered as exponentially distributed while repair and maintenance time distributions are considered as arbitrary. All random variables are statistically independent. Repairs are perfect. Various measures of system effectiveness such as transition probabilities, mean sojourn times, mean time to system failure, steady state availability, busy period of the server due to replacements, repair and preventive maintenance, expected number of repairs, replacements and preventive maintenance, expected number of visits by the server and expected profit earned by the system in $(0, t)$ are obtained by using the technique of semi-Markov process and RPT. To highlight the importance of the study numerical results are also obtained for MTSF, availability and profit function.

\section{Model Description}

(i) Initially system consists of two identical units- one operative and other is kept as cold standby.

(ii) Both units have three modes- normal, under repair due to failure and under preventive maintenance.

(iii) The failed unit is replaced by new one if the repair of the unit is not possible up to a maximum repair time.

(iv)The failure and maximum operation time distribution are exponentially distributed while repair and preventive maintenance times are distributed arbitrarily.

(v) There is a single server who visits the system immediately as and when required.

(vi)The switch devices, repairs and preventive maintenance are perfect.

(vii)All random variables are statistically independent. 


\section{Notations}

$\mathrm{O}$

Cs

$\Lambda$

$\alpha_{0}$

$\beta_{0}$

$\mathrm{f}(\mathrm{t}) / \mathrm{F}(\mathrm{t})$

$\mathrm{h}(\mathrm{t}) / \mathrm{H}(\mathrm{t})$

$\mathrm{Pm} / \mathrm{PM}$

FUr/FUR

FWr / FWR

Furp/FURP

$\mathrm{g}(\mathrm{t}) / \mathrm{G}(\mathrm{t})$

$\mathrm{q}_{\mathrm{ij}}(\mathrm{t}) / \mathrm{Q}_{\mathrm{ij}}(\mathrm{t})$

$\mathrm{q}_{\mathrm{ij} . \mathrm{k}}(\mathrm{t}) /$

$\mathrm{Q}_{\mathrm{ij} . \mathrm{k}}(\mathrm{t})$

$\mathrm{Mi}_{\mathrm{i}}(\mathrm{t})$

pdf / cdf

$\mathrm{Wi}(\mathrm{t})$

$\mathrm{m}_{\mathrm{ij}}$

$\mu_{i}$

(S) / C

$\sim$ /
The unit is operative and in normal mode,

The unit is in cold standby,

Constant failure rate of the unit,

Constant rate of Maximum Operation Time,

Constant rate of Maximum Repair Time,

pdf / cdf of preventive maintenance of the unit after maximum operation time, pdf / cdf of replacement time of the unit,

The unit is under preventive Maintenance/ under preventive maintenance continuously from previous state,

The unit is failed and is under repair / under repair continuously from previous state,

The unit is failed and is waiting for repair/ waiting for repair from previous state,

The unit is failed and is under replacement / under replacement continuously from previous state,

$\mathrm{pdf}$ / cdf of repair time of the failed unit,

$\mathrm{pdf} / \mathrm{cdf}$ of direct transition time from a regenerative state i to a regenerative state $\mathrm{j}$ without visiting any other regenerative state,

$\mathrm{pdf} / \mathrm{cdf}$ of first passage time from a regenerative state i to a regenerative state $j$ or to a failed state $j$ visiting state $k$ once in $(0, t]$,

Probability that the system is up initially in state $S_{i} \in E$ is up at time ' $t$ ' without visiting to any other regenerative state,

Probability density function/ Cumulative density function,

Probability that the server is busy in state $S_{i}$ up to time $t$ without making transition to any other regenerative state or returning to the same via one or more non regenerative states,

Contribution to mean sojourn time in state $S_{\mathrm{i}}$ when system transits directly to state $S_{j}\left(S_{i}, S_{j} \in E\right)$ so that $\mu_{i}=\sum_{j} m_{i j}$ where $m_{i j}=\int t d Q_{i j}(t)=-q_{i j}^{*}$ and $\mu_{\mathrm{i}}$ is the mean sojourn time in state $S_{\mathrm{i}} \in \mathrm{E}$,

The mean Sojourn time in state $S_{i}$ this is given by

$\mu_{i}=E\left(T_{i}\right)=\int_{0}^{\infty} P\left(T_{i}>t\right) d t=\sum_{j} m_{i j}$, where $T_{i}$ is the sojourn time in state $\mathrm{S}_{\mathrm{i}}$,

Symbol for Stieltjes convolution / Laplace convolution,

Symbol for Laplace Stieltjes Transform (LST) / Laplace Transform (LT).

In view of the above notations and assumptions the system may be in one of the following states:

$S_{0}=(O, C s), S_{1}=(O, P m), S_{2}=(O, F u r), S_{3}=(F U R, F w r), S_{4}=($ Furp,$F W R)$

$S_{5}=(O$, Furp $), S_{6}=(F U R P, F w r)$ and $S_{7}=(P M, F w r)$

Up state are: $S_{0}, S_{1}, S_{2}, S_{5}$

Down states are: $S_{3}, S_{4}, S_{6}, S_{7}$

Regenerative states: $S_{0}, S_{1}, S_{2}, S_{5} \quad$ Non-regenerative states: $S_{3}, S_{4}, S_{6}, S_{7}$

\section{Transition Probabilities and Mean Sojourn Times:}

Simple probabilistic considerations yield the following expressions for the non-zero elements

$$
p_{i j}=Q_{i}(\infty)=\int_{0}^{\infty} q_{i}(t) d
$$


as

$$
\begin{aligned}
& P_{01}=\frac{\alpha_{0}}{\alpha_{0}+\lambda}, P_{02}=\frac{\lambda}{\alpha_{0}+\lambda}, P_{10}=\frac{\alpha}{\alpha+\lambda}, P_{17}=\frac{\lambda}{\alpha+\lambda}, P_{25}=\frac{\beta_{0}}{\beta_{0}+\theta+\lambda}, P_{23}=\frac{\lambda}{\beta_{0}+\lambda+\theta}, \\
& P_{20}=g^{*}\left(\lambda+\beta_{0}\right), P_{34}=1, P_{42}=1, P_{62}=1, P_{72}=1, P_{50}=h^{*}\left(\lambda_{1}\right), P_{56}=\left[1-h^{*}(\lambda)\right], \\
& P_{22.3 .4}=\left[1-g^{*}\left(\beta_{0}\right)\right], P_{12.7}=\left[1-f^{*}(\lambda)\right], P_{52.6}=\left[1-h^{*}(\lambda)\right] .
\end{aligned}
$$

It can be easily verified that

$$
\begin{aligned}
& P_{01}+P_{02}=P_{10}+P_{17}=P_{20}+P_{25}+P_{23}=P_{50}+P_{56}=P_{34}=P_{42}=P_{62}=P_{72}=P_{22.3,4}+P_{25}+P_{20}= \\
& P_{12.7}+P_{10}=P_{52.6}+P_{50}=1
\end{aligned}
$$

The mean sojourn times $\left(\mu_{\mathrm{i}}\right)$ of the state $S_{i}$ are:

$$
\begin{aligned}
& \mu_{0}=\frac{1}{\lambda+\alpha_{0}}, \mu_{1}=\frac{1}{\alpha+\lambda}, \mu_{2}=\frac{1}{\beta+\theta+\lambda}, \mu_{5}=\frac{1}{\lambda+\beta}, \mu_{1}^{\prime}=\frac{1}{\alpha}, \mu_{5}^{\prime}=\frac{1}{\beta}, \\
& \mu_{2}^{\prime}=\frac{\lambda+\beta-(\lambda \beta)}{\beta(\beta+\lambda+\theta)}
\end{aligned}
$$

\section{Reliability and Mean Time to System Failure (MTSF)}

Let $\varphi_{i}(t)$ be the cdf of first passage time from the regenerative state $S_{i}$ to a failed state. Regarding the failed state as absorbing state, we have the following recursive relations for $\varphi_{i}(t)$ :

$$
\varphi_{i}(t)=\sum_{j} Q_{i, j}(t) \circledR \varphi_{j}(t)+\sum_{k} Q_{i, k}(t),
$$

where $S_{j}$ is an un-failed regenerative state to which the given regenerative state $S_{i}$ can transit and $S_{k}$ is a failed state to which the state $S_{i}$ can transit directly. Taking LST of Eq. (5) and solving for $\tilde{\phi}_{0}(s)$, we have

$$
R^{*}(s)=\frac{1-\tilde{\varphi}_{0}(s)}{s} .
$$

The reliability of the system model can be obtained by taking inverse Laplace transform of Eq. (6). The mean time to system failure (MTSF) is given by

$$
\begin{aligned}
& \text { MTSF }=\lim _{s \rightarrow o} \frac{1-\tilde{\phi}_{0}(s)}{s}=\frac{N_{1}}{D_{1}} \text { where } \\
& N_{1}=\mu_{0}+\mu_{2} p_{02}+\mu_{1} p_{01}+p_{02} p_{25} \mu_{5} \text { and } D_{1}=1-p_{02} p_{02}-p_{50} p_{25} p_{02}-p_{01} p_{10}
\end{aligned}
$$

\section{Steady State Availability}

Let $A_{i}(t)$ be the probability that the system is in up-state at instant 't' given that the system entered regenerative state $S_{i}$ at $\mathrm{t}=0$. The recursive relations for $\mathrm{A}_{\mathrm{i}}(\mathrm{t})$ are given as

$$
A_{i}(t)=M_{i}(t)+\sum_{j} q_{i, j}^{(n)}(t) \Subset A_{j}(t)
$$


where $S_{j}$ is any successive regenerative state to which the regenerative state $S_{i}$ can transit through $n$ transitions. $M_{i}(t)$ is the probability that the system is up initially in state $S_{i} \in E$ up at time $t$ without visiting to any other regenerative state, we have

$$
\begin{aligned}
& M_{0}(t)=e^{-\left(\lambda+\alpha_{0}\right) t}, M_{1}(t)=e^{-\left(\lambda+\alpha_{0}\right) t} \overline{F(t)}, M_{2}(t)=e^{-\left(\lambda+\alpha_{0}\right) t} \overline{H(t)}, M_{4}(t)=e^{-\left(\lambda+\alpha_{0}\right) t \overline{G(t)}} \\
& M_{3}(t)=e^{-\left(\lambda+\alpha_{0}\right) t \overline{M(t)}}
\end{aligned}
$$

Taking LT from Eqs. (8) and solving for $A_{0}^{*}(s)$, the steady state availability is given by

$$
\begin{aligned}
& A_{0}(\infty)=\lim _{s \rightarrow 0} s A_{0}^{*}(s)=\frac{N_{2}}{D_{2}} \text {, where } \\
& N_{2}=\left[\left(\mu_{0}+\mu_{1} p_{01}\right)\left(1-p_{22.3 .4}-p_{52.6} p_{25}\right)\right]+\left[\left(\mu_{2}+\mu_{5} p_{25}\right)\left(p_{02}+p_{12.7} p_{01}\right)\right] \text { and } \\
& D_{2}=\left[\left(\mu_{0}+\mu_{1}^{\prime} p_{01}\right)\left(1-p_{22.3 .4}-p_{52.6} p_{25}\right)\right]+\left[\left(\mu_{2}^{\prime}+\mu_{5}^{\prime} p_{25}\right)\left(p_{02}+p_{12.1} p_{01}\right)\right]
\end{aligned}
$$

\section{Busy Period Analysis of the Server}

(a) Due to Preventive Maintenance (PM)

Let $B_{i}^{P}(t)$ be the probability that the server is busy in preventive maintenance of the system (unit) at an instant ' $\mathrm{t}$ ' given that the system entered state $S_{i}$ at $\mathrm{t}=0$. The recursive relations for $B_{i}^{P}(t)$ are as follows:

$$
B_{i}^{p}(t)=W_{i}(t)+\sum_{j} q_{i, j}^{(n)}(t) \subseteq B_{j}^{p}(t),
$$

where $S_{j}$ is any successive regenerative state to which the regenerative state $S_{i}$ can transit through $n$ transitions. $\mathrm{W}_{\mathrm{i}}(\mathrm{t})$ be the probability that the server is busy in state $\mathrm{S}_{\mathrm{i}}$ due to preventive maintenance up to time $t$ without making any transition to any other regenerative state or returning to the same via one or more non-regenerative states and so

$W_{1}(t)=e^{-\left(\lambda+\alpha_{0}\right) t} \bar{F}(t)+\left(\alpha_{0} e^{-\left(\lambda+\alpha_{0}\right) t} \Subset 1\right) \bar{F}(t)+\left(\lambda e^{-\left(\lambda+\alpha_{0}\right) t}(1) \bar{F}(t)\right.$

\section{(b) Due to Repair}

Let $B_{i}^{R}(t)$ be the probability that the server is busy in repairing the unit due to failure at an instant ' $\mathrm{t}$ ' given that the system entered state $S_{i}$ at $\mathrm{t}=0$. The recursive relations for $B_{i}^{R}(t)$ are as follows:

$$
B_{i}^{R}(t)=W_{i}(t)+\sum_{j} q_{i, j}^{(n)}(t) \subseteq B_{j}^{R}(t),
$$

where $S_{j}$ is any successive regenerative state to which the regenerative state $S_{i}$ can transit through $n$ transitions. $\mathrm{W}_{\mathrm{i}}(\mathrm{t})$ be the probability that the server is busy in state $\mathrm{S}_{\mathrm{i}}$ due to repair of the unit up to time $\mathrm{t}$ without making any transition to any other regenerative state or returning to the same via one or more non-regenerative states and so

$$
W_{2}=e^{-\left(\lambda+\beta_{0}\right) t} \bar{G}(t)++\left(\beta_{0} e^{-\left(\lambda+\beta_{0}\right) t} \odot 1\right) \bar{G}(t)+\left(\lambda e^{-\left(\lambda+\beta_{0}\right) t} \odot 1\right) \bar{G}(t)
$$

(c) Due to Replacement 
Let $\mathrm{B}_{\mathrm{i}}^{\mathrm{Rp}}(\mathrm{t})$ be the probability that the server is busy in the replacement of the unit due to failure at an instant ' $\mathrm{t}$ ' given that the system entered state $S_{i}$ at $\mathrm{t}=0$. The recursive relations for $\mathrm{B}_{\mathrm{i}}^{\mathrm{Rp}}(\mathrm{t})$ are as follows:

$$
B_{i}^{R p}(t)=W_{i}(t)+\sum_{j} q_{i, j}^{(n)}(t) \subset B_{j}^{R p}(t),
$$

where $S_{j}$ is any successive regenerative state to which the regenerative state $S_{i}$ can transit through $n$ transitions. $\mathrm{W}_{\mathrm{i}}(\mathrm{t})$ be the probability that the server is busy in state $\mathrm{S}_{\mathrm{i}}$ due to repair of the unit up to time $\mathrm{t}$ without making any transition to any other regenerative state or returning to the same via one or more non-regenerative states and so

$W_{5}=e^{-\lambda t} \bar{H}(t)$

Taking LT of above relations and solving for $B_{0}^{*^{P}}(\mathrm{~s}), B_{0}^{*_{R}}(\mathrm{~s})$ and $\mathrm{B}_{0}^{{ }^{\mathrm{Rp}}}(\mathrm{s})$. The time for which server is busy due to preventive maintenance, repair and replacement is

$$
B_{0}^{P}=\lim _{s \rightarrow 0} s B_{0}^{* P}(s)=\frac{N_{3}^{P}}{D_{2}}, \mathrm{~B}_{0}^{\mathrm{R}}=\lim _{\mathrm{s} \rightarrow 0} \mathrm{sB}_{0}^{* \mathrm{R}}(\mathrm{s})=\frac{\mathrm{N}_{3}^{\mathrm{R}}}{\mathrm{D}_{2}} \text { and } \mathrm{B}_{0}^{\mathrm{Rp}}=\lim _{\mathrm{s} \rightarrow 0} \mathrm{sB}_{0}^{* \mathrm{Rp}}(\mathrm{s})=\frac{\mathrm{N}_{3}^{\mathrm{Rp}}}{\mathrm{D}_{2}}
$$

where

$$
\begin{aligned}
& N_{3}{ }^{p}=W_{1}^{*}(0)\left(-p_{01}\right)\left(1-p_{22.3 .4}-p_{25} p_{52.6}\right), N_{3}{ }^{R}=W^{*}(0)\left[p_{01} p_{12.7}+p_{02}\right], \\
& N_{3}{ }^{R P}=\left(W_{2}^{r p}(0)+W_{5}^{*}(0) p_{25}\right)\left(p_{02}+p_{01} p_{127}\right) \text { and } D_{2} \text { is already defined. }
\end{aligned}
$$

\section{Expected Number of Preventive Maintenances}

Let $R_{i}^{p}(t)$ be the expected number of preventive maintenances conducted by the server in $(0, \mathrm{t}]$ given that the system entered the regenerative state $S_{i}$ at t $=0$. The recursive relations for $R_{i}^{p}(t)$ are given as

$$
R_{i}^{p}(t)=\sum_{j} Q_{i, j}^{(n)}(t) \circledR\left[\delta_{j}+R_{j}^{p}(t)\right],
$$

where $S_{j}$ is any regenerative state to which the given regenerative state $S_{i}$ transits and $\delta j=1$, if $S_{j}$ is the regenerative state where the server does job afresh, otherwise $\delta j=0$. Taking LST of Eqs. (15) and solving for $\tilde{R}_{0}^{p}(s)$. The expected numbers of preventive maintenances per unit time are given by

$$
R_{0}^{p}(\infty)=\lim _{s \rightarrow 0} s \tilde{R}_{0}^{p}(s)=\frac{N_{4}^{p}}{D_{2}}
$$

where $N_{4}{ }^{P}=\left(p_{01}+p_{12.7}\right)\left(-p_{01}\right)\left(1-p_{22.3 .4}-p_{25} p_{52.6}\right)$ and $D_{2}$ is already mentioned.

\section{Expected Number of Repairs}

Let $R_{i}^{r}(t)$ be the expected number of repairs by the server in $(0, \mathrm{t}]$ given that the system entered the regenerative state $S_{i}$ at $\mathrm{t}=0$. The recursive relationships for $R_{i}^{r}(t)$ are given as

$$
R_{i}^{r}(t)=\sum_{j} Q_{i, j}^{(n)}(t) \circledR\left[\delta_{j}+R_{j}^{r}(t)\right],
$$


where $S_{j}$ is any regenerative state to which the given regenerative state $S_{i}$ transits and $\delta j=1$, if $S_{j}$ is the regenerative state where the server does job afresh, otherwise $\delta j=0$. Taking LST of Eqs. (17) and solving for $\tilde{R}_{0}^{r}(s)$. The expected numbers of repairs per unit time are given by

$$
R_{0}^{r}(\infty)=\lim _{s \rightarrow 0} s \tilde{R}_{0}^{r}(s)=\frac{N_{4}^{r}}{D_{2}},
$$

where $N_{4}{ }^{R}=\left(p_{20}+p_{22.3 .4}\right)\left(p_{01} p_{12.7}+p_{02}\right)$ and $\mathrm{D}_{2}$ is already mentioned.

\section{Expected Number of Visits by the Server}

Let $N_{i}(t)$ be the expected number of visits by the server in $(0, \mathrm{t}]$ given that the system entered the regenerative state $S_{i}$ at $\mathrm{t}=0$. The recursive relations for $N_{i}(t)$ are given as

$$
N_{i}(t)=\sum_{j} Q_{i, j}^{(n)}(t) \circledR\left[\delta_{j}+N_{i}(t)\right],
$$

where $S_{j}$ is any regenerative state to which the given regenerative state $S_{i}$ transits and $\delta j=1$, if $j$ is the regenerative state where the server does job afresh, otherwise $\delta j=0$. Taking LST of Eqs. (19) and solving for $\tilde{R}_{0}^{I}(s)$, the expected numbers of replacements per unit time are given by

$$
N_{0}(\infty)=\lim _{s \rightarrow 0} s \tilde{N}_{0}(s)=\frac{N_{5}}{D_{2}}
$$

where $N_{0}=\left(p_{01}+p_{02}\right)\left(1-p_{22.3 .4}-p_{25} p_{52.6}\right)$ and $\mathrm{D}_{2}$ is already mentioned.

\section{Expected number of replacements}

Let $R_{i}^{r p}(t)$ be the expected number of replacements by the server in $(0, \mathrm{t}]$ given that the system entered the regenerative state $S_{i}$ at $\mathrm{t}=0$. The recursive relations for $R_{i}^{r p}(t)$ are given as

$$
R_{i}^{r p}(t)=\sum_{j} Q_{i, j}^{(n)}(t) \circledR\left[\delta_{j}+R_{j}^{r p}(t)\right],
$$

where $S_{j}$ is any regenerative state to which the given regenerative state $S_{i}$ transits and $\delta j=1$, if $S_{j}$ is the regenerative state where the server does job afresh, otherwise $\delta j=0$. Taking LST of Eqs. (21) and solving for $\tilde{R}_{0}^{r}(s)$, The expected numbers of repairs per unit time are given by

$$
\begin{aligned}
& R_{0}^{r}(\infty)=\lim _{s \rightarrow 0} s \tilde{R}_{0}^{r}(s)=\frac{N_{4}^{r}}{D_{2}} \\
& N_{4}{ }^{R P}=\left(p_{22.3 .4}+p_{25} p_{52.6}+p_{25} p_{50}\right)\left(p_{02}+p_{12.7} p_{01}\right) \text { and } \mathrm{D}_{2} \text { is already mentioned. }
\end{aligned}
$$

\section{Profit Analysis}

The profit incurred to the system model in steady state can be obtained as

$$
P=K_{0} A_{0}-K_{1} B_{0}^{R}-K_{2} B_{0}^{P}-K_{3} R_{0}^{R}-K_{4} R_{0}^{P}-K_{5} N_{0}-K_{6} R_{0}^{R P}
$$

where

$\mathrm{K}_{0}=$ Revenue per unit up-time of the system

$\mathrm{K}_{1}=$ Cost per unit time for which server is busy due to repair

$\mathrm{K}_{3}=$ Cost per unit time repair of the unit

$\mathrm{K}_{4}=$ Cost per unit time preventive maintenance of the unit 
$\mathrm{K}_{2}=$ Cost per unit time for which server is busy $\mathrm{K}_{5}=$ Cost per unit time visit by the server due to preventive maintenance

$K_{6}=$ cost per unit time replacement of unit

\section{Conclusion}

The graphical results for various reliability measures are obtained in Figs. 1-3 by considering the particular case $g(t)=\theta e^{-\theta t}, \mathrm{~h}(\mathrm{t})=\beta \mathrm{e}^{-\beta \mathrm{t}}$ and $f(t)=\alpha e^{-\alpha t}$ with cost $\mathrm{K}_{0}=5000, \mathrm{~K}_{1}=250, \mathrm{~K}_{2}=300$, $K_{3}=200, K_{4}=190, K_{5}=210, K_{6}=160$ and $K_{0}=150$. From Figs. 1-3, it is revealed that MTSF, profit and availability decrease with the increase of failure rate $(\lambda)$ and maximum operation time $\left(\alpha_{0}\right)$, but the value of steady state availability and MTSF increase with the increase of repair rate $(\theta)$ and preventive maintenance rate $(\alpha)$. The profit of the system declines with the increases of preventive maintenance rate $(\alpha)$. Thus finally it is concluded that a cod standby system in which a maximum repair time is given to server for repair can be made more reliable and profitable to use

(i) By taking one more unit in cold standby,

(ii) By ignoring the concept of priority to operation over preventive maintenance,

(iii) By increasing the repair rate,

(iv) By increasing the maximum repair time.

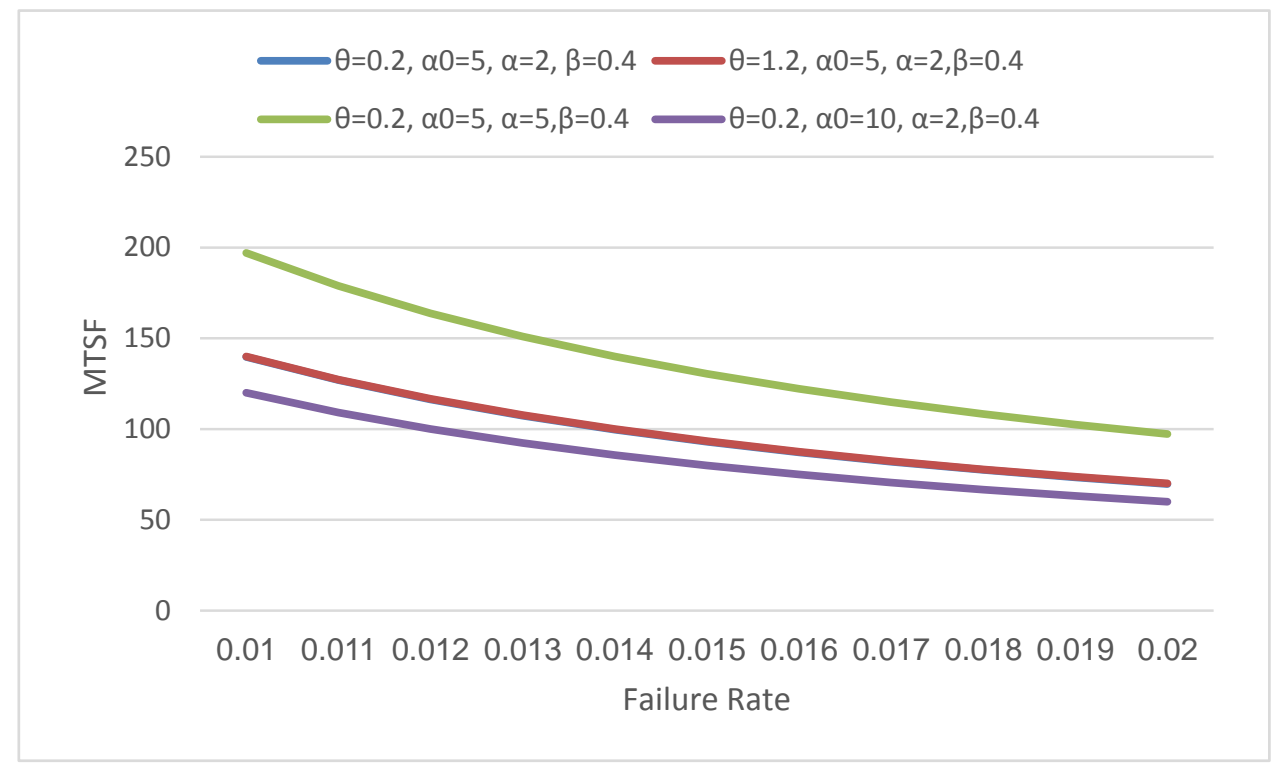

Fig. 1. MTSF vs. Failure Rate 


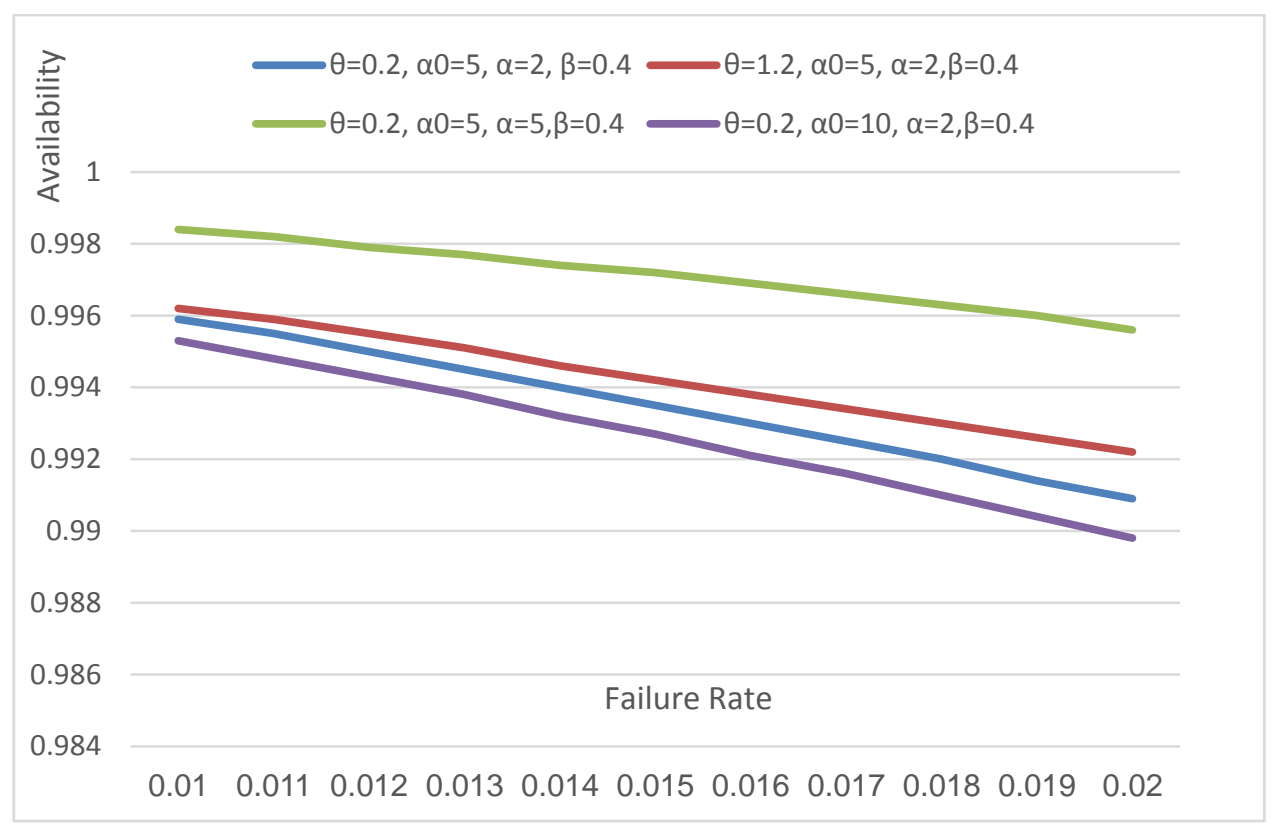

Fig. 2. Availability vs. Failure Rate

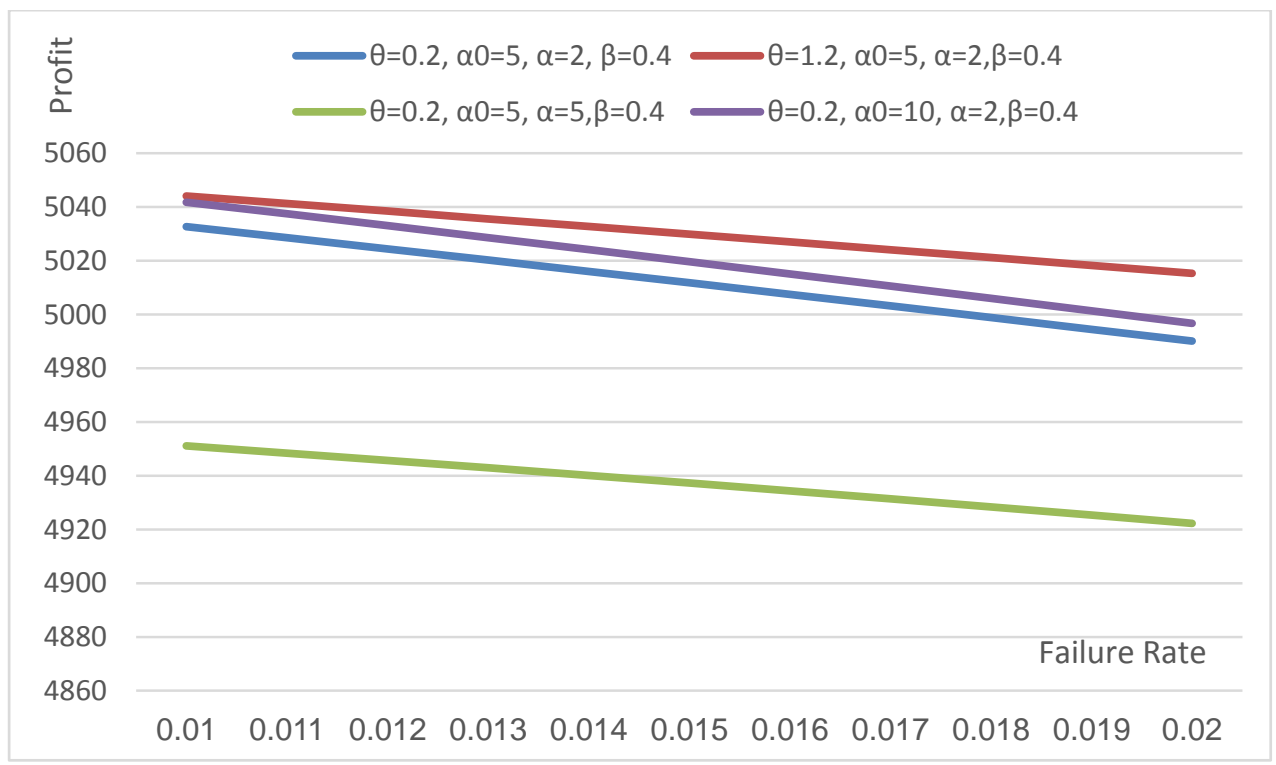

Fig. 3. Profit vs. Failure Rate

\section{References}

Barak, A. K., \& Barak, M. S. (2013). Reliability modelling of a cloud with arrival time of the server subject to maximum operation and repair times. Indian Journal of Science, 3(6), 6-10.

Barak, A., \& Malik, S. C. (2014). Reliability Measure of a Standby System with Arrival Time of the Server Subject to Maximum Operation and Repair Time. IJSSST, 14(5), 55-62.

Cao, J., \& Wu, Y. (1989). Reliability analysis of a two-unit cold standby system with a replaceable repair facility. Microelectronics Reliability, 29(2), 145-150.

Chander, S. (2005). Reliability models with priority for operation and repair with arrival time of the server. Pure and Applied Mathematika Sciences, 61(1-2), 9-22.

Goel, L. R., \& Sharma, S. C. (1989). Stochastic analysis of a 2-unit standby system with two failure modes and slow switch. Microelectronics Reliability, 29(4), 493-498. 
Gopalan, M. N., \& Nagarwalla, H. E. (1985). Cost-benefit analysis of a one-server two-unit cold standby system with repair and preventive maintenance. Microelectronics Reliability, 25(2), 267269.

Gopalan, M. N., \& Bhanu, K. S. (1995). Cost analysis of a two unit repairable system subject to online preventive maintenance and/or repair. Microelectronics Reliability, 35(2), 251-258.

Jin, X., Li, L., \& Ni, J. (2009). Option model for joint production and preventive maintenance system. International Journal of Production Economics, 119(2), 347-353.

Kumar, A., \& Malik, S. C. (2012). Reliability modeling of a computer system with priority to s/w replacement over h/w replacement subject to MOT and MRT. International Journal of Pure and Applied Mathematics, 80(5), 693-709.

Kumar, A., \& Malik, S. C. (2012). Stochastic modeling of a computer system with priority to PM over S/W replacement subject to maximum operation and repair Times. International Journal of Computer Applications, 43(3), 27-34.

Kumar, A., Malik, S. C., \& Barak, M. S. (2012). Reliability modelling of a computer system with independent $\mathrm{H} / \mathrm{W}$ and $\mathrm{S} / \mathrm{W}$ failures subject to maximum operation and repair times. International Journal of Mathematical Archive (IJMA) ISSN 2229-5046, 3(7).

Kumar, A., \& Malik, S. (2015). Reliability measures of a computer system with priority to PM over the H/W repair activities subject to MOT and MRT. Management Science Letters, 5(1), 29-38.

Malik, S. C., \& Nandal, P. (2010). Cost-analysis of stochastic models with priority to repair over preventive maintenance subject to maximum operation time, edited book, learning manual on modeling, optimization and their applications. Optimization and Their Applications.

Kumar, A., \& Malik, S. C. (2011). Profit analysis of a computer system with Priority to software replacement over hardware repair subject to maximum operation and repair times. International Journal of Engineering Science and Technology (IJEST), 3(10), 7452-7468.

Malik, S. C. \& Gitanjali (2012). Cost-benefit analysis of a parallel system with arrival time of the server and maximum repair time. International Journal of Computer Applications, 46(5),39-44.

Malik, S. C., \& Munday, V. J. (2014). Stochastic modelling of a computer system with hardware redundancy. International Journal of Computer Applications, 89(7), 26-30.

Mahmoud, M. A. W., \& Moshref, M. E. (2010). On a two-unit cold standby system considering hardware, human error failures and preventive maintenance. Mathematical and Computer Modelling, 51(5), 736-745.

Mokaddis, G. S., El-Sherbeny, M. S., \& Al-Esayeh, E. (2008). Human error and partial hardware failure modeling of parallel and standby redundant system. The Journal of Mathematics \& Computer Science, 19(2), 141-152.

Mokaddis, G. S., El-Sherbeny, M. S., \& Al-Esayeh, E. (2008). A dissimilar two-unit parallel system with common-cause failure and preventive maintenance. The Journal of Mathematics \& Computer Science, 19(2), 153-167.

Moghaddass, R., Zuo, M. J., \& Qu, J. (2011). Reliability and availability analysis of a repairable-outof-system with repairmen subject to shut-off rules. Reliability, IEEE Transactions on, 60(3), 658666.

Osaki, S., \& Asakura, T. (1970). A two-unit standby redundant system with repair and preventive maintenance. Journal of Applied Probability, 641-648.

Kapur, P. K., \& Kapoor, K. R. (1974). A two-unit warm standby system with repair and preventive maintenance. Indian Journal Pure Applied Mathematics (pp. 13-27).

Singh, S. K., \& Agrafiotis, G. K. (1995). Stochastic analysis of a two-unit cold standby system subject to maximum operation and repair time. Microelectronics Reliability, 35(12), 1489-1493.

Wu, Q., \& Wu, S. (2011). Reliability analysis of two-unit cold standby repairable systems under Poisson shocks. Applied Mathematics and Computation, 218(1), 171-182.

Chandrasekhar, P., Natarajan, R., \& Yadavalli, V. S. S. (2004). A study on a two unit standby system with Erlangian repair time. Asia-Pacific Journal of Operational Research, 21(03), 271-277.

Zhang, Y. L., \& Wang, G. J. (2009). A geometric process repair model for a repairable cold standby system with priority in use and repair. Reliability Engineering \& System Safety, 94(11), 1782-1787. 